\title{
Somatotopic Organization of Gentle Touch Processing in the Posterior Insular Cortex
}

\author{
Malin Björnsdotter, Line Löken, Håkan Olausson, Åke Vallbo, and Johan Wessberg \\ Institute of Neuroscience and Physiology, University of Gothenburg, 41390 Göteborg, Sweden
}

A network of thin ( $\mathrm{C}$ and $\mathrm{A} \delta$ ) afferents relays various signals related to the physiological condition of the body, including sensations of gentle touch, pain, and temperature changes. Such afferents project to the insular cortex, where a somatotopic organization of responses to noxious and cooling stimuli was recently observed. To explore the possibility of a corresponding body-map topography in relation to gentle touch mediated through $\mathrm{C}$ tactile (CT) fibers, we applied soft brush stimuli to the right forearm and thigh of a patient (GL) lacking $\mathrm{A} \beta$ afferents, and six healthy subjects during functional magnetic resonance imaging (fMRI). For improved fMRI analysis, we used a highly sensitive multivariate voxel clustering approach. A somatotopic organization of the left (contralateral) posterior insular cortex was consistently demonstrated in all subjects, including GL, with forearm projecting anterior to thigh stimulation. Also, despite denying any sense of touch in daily life, GL correctly localized $97 \%$ of the stimuli to the forearm or thigh in a forced-choice paradigm. The consistency in activation patterns across GL and the healthy subjects suggests that the identified organization reflects the central projection of CT fibers. Moreover, substantial similarities of the presently observed insular activation with that described for noxious and cooling stimuli solidify the hypothesized sensory-affective role of the CT system in the maintenance of physical well-being as part of a thin-afferent homeostatic network.

\section{Introduction}

Cutaneous small-diameter $(\mathrm{C}$ and $\mathrm{A} \delta$ ) afferents mediate various information related to the physiological condition of the body, including sensations of pain, temperature, itch, and gentle touch (for review, see Craig, 2002). Extensive studies suggest that thinfiber networks mediating pain and temperature connect from the superficial laminae of the dorsal horn via somatotopically arranged projections through the spinothalamic tract (STT) to a specific ventromedial posterior thalamic nuclei (VMPo), terminating in the contra-lateral posterior insular cortex (Craig et al., 1994, 1999, 2000; Blomqvist and Craig, 2000; Craig, 2003; Craig and Zhang, 2006) (but see Willis et al., 2002). Consistent with these projections, functional imaging of innocuous cooling as well as painful stimuli have revealed a cortically maintained somatotopic organization of the posterior insula with upper body afferents activating regions anterior to those of the lower body (Brooks et al., 2005; Hua le et al., 2005; Henderson et al., 2007).

The anatomical organization of the mechanoreceptive $\mathrm{C}$ tactile (CT) afferent system responding to gentle touch is, however, inadequately understood (Vallbo et al., 1999; Olausson et al., 2002). CT afferents, identified in a variety of human skin areas, including the forehead, forearm, thigh, and lower leg (Nordin, 1990; Vallbo et al., 1999; Edin, 2001; Löken et al., 2007), are

Received Jan. 22, 2009; revised May 18, 2009; accepted June 20, 2009.

This study was supported by the Swedish Research Council (Grant K2007-63X-3548) and the Sahlgrenska University Hospital (Grant ALFGBG 3161). We are grateful to GL for her continuous enthusiastic participation and to Dr. M.C. Bushnell for her contribution in the examination of $\mathrm{GL}$.

Correspondence should be addressed to Malin Björnsdotter, Medicinaregatan 11, 41390 Göteborg, Sweden. E-mail: malin.bjornsdotter@neuro.gu.se.

DOI:10.1523/JNEUROSCI.0400-09.2009

Copyright $\odot 2009$ Society for Neuroscience $\quad$ 0270-6474/09/299314-07\$15.00/0 highly sensitive to soft, slow skin deformations (Zotterman, 1939; Douglas and Ritchie, 1957; Iggo and Muir, 1969; Kumazawa and Perl, 1977; Nordin, 1990; Vallbo et al., 1993, 1999; Edin, 2001). Such stimulation of the skin invariably coactivates thick, myelinated fibers, preventing the selective study of CT afferents in healthy subjects. CT system physiology has, nevertheless, been successfully explored in a patient (GL) with neuronopathy syndrome (Sterman et al., 1980), who lacks large myelinated afferents but whose C fibers are intact (Olausson et al., 2002, 2008a). Despite asserting no touch sensation below the level of the nose in daily life, GL can, under focused concentration, detect the soft stroking of a brush and reports a vague pleasant sensation in response to the stimuli. Similarly, we recently demonstrated that neurologically intact individuals' perception of tactile pleasantness is correlated to CT-but not to $\mathrm{A} \beta$ - activation (Löken et al., 2009).

Functional imaging in GL and a similarly deafferented subject (IW) revealed that CT stimulation activates the contralateral posterior insular cortex (Olausson et al., 2002, 2008b), consistent with other thin-fiber mediated sensations such as pain and temperature. No activity in somatosensory cortices was observed, however, suggesting differential central projection networks in addition to the functional dissociation between the CT and A $\beta$ afferent systems.

Surprisingly, we recently observed that GL can not only detect a soft brush stimulus, but also distinguish the body quadrant to which it was applied at an accuracy of $72 \%$ (Olausson et al., 2008a). GL's performance, although severely worse than that of healthy subjects who correctly identify all stimuli without exception, suggests that the CT system projects some, albeit crude, information regarding stimulus location. 
This study consequently explores whether CT mediated touch projects to the posterior insular cortex in a somatotopic manner similar to that identified for cooling and noxious stimuli. To this end, we used functional magnetic resonance imaging (fMRI) and a highly sensitive multivariate analysis approach (Björnsdotter Åberg and Wessberg, 2008).

\section{Materials and Methods}

Participants. The Ethical Review Board at the University of Gothenburg approved the study, and the experiments were performed in accordance with the Declaration of Helsinki. Informed consent was obtained from one subject (GL, age 56, right handed, female) with sensory neuronopathy syndrome (Sterman et al., 1980) and from six healthy subjects (aged 22-28 years, all right handed, three male).

At the age of 31, GL suffered permanent specific loss of large-diameter myelinated afferents, leaving unmyelinated and small-diameter myelinated afferents intact (Forget and Lamarre, 1995). Motor nerve conduction and electromyography findings are within the healthy range, and thresholds for temperature and pain detection are largely normal (Olausson et al., 2002, 2008a). GL routinely denies any ability to identify or localize touch below the level of the nose (Forget and Lamarre, 1995). In a forced choice task she did, however, demonstrate the perception of gentle touch in the hairy but not in the glabrous skin (lacking CT afferents), whereas she failed to detect vibratory stimuli (which poorly excite CT afferents) in both types of skin (Olausson et al., 2008a). Moreover, in a four-alternative forced choice procedure, she identified $72 \%$ of soft brush stimuli to the correct extremity (at chance level of 25\%). Healthy subjects, in contrast, detect gentle touch as well as vibration without fail in both glabrous and hairy skin, and can localize point indentation on the hairy skin to an accuracy in the range of $2 \mathrm{~cm}$ (Norrsell and Olausson, 1994).

Stimuli. Gentle stimulation, known to vigorously activate CT afferents in humans as well as in other species (Zotterman, 1939; Douglas and Ritchie, 1957; Bessou et al., 1971; Kumazawa and Perl, 1977; Nordin, 1990; Vallbo et al., 1993, 1999; Edin, 2001), was delivered using a 7-cmwide soft artist's goat hair brush. The experimenter manually stroked the brush on the right forearm or thigh in a proximal to distal direction, using an indentation force of $0.8 \mathrm{~N}$. The brushing velocity varied in the range $4-7.5 \mathrm{~cm} / \mathrm{s}$ (see Psychophysical testing and fMRI experimental design for details on the duration and distance covered).

Psychophysical testing. We performed a two-alternative forced choice test where GL was instructed to report whether the brush stimulation was applied to the right forearm or to the right thigh. Brush strokes were applied manually for a duration of $4 \mathrm{~s}$ across a distance of $16 \mathrm{~cm}$, and 32 trials were performed in a pseudo-random sequence. Visual cues were prevented by fabric mounted such that the experimenter and brush were shielded, and GL was wearing earplugs.

fMRI experimental design. GL and the healthy subjects were studied in different laboratories (Montreal, QC, Canada and Göteborg, Sweden) with slight variations in protocol. The experimenter applied the tactile stimulation according to cues from the scanner, and all subjects were instructed to focus on the stimulus throughout the fMRI scanning session. In the healthy subjects, the distance covered was $16 \mathrm{~cm}$ for a duration of $3 \mathrm{~s}$, whereas in the case of GL the distance was $30 \mathrm{~cm}$ and the duration $4 \mathrm{~s}$. Three-volume blocks of forearm brushing, thigh brushing, or no brushing (rest) were alternated in a pseudo-random order with equal numbers of each of the three conditions. The condition order remained fixed throughout each scan and across participants, and the scanning session consisted of one anatomical and six functional scans. During each functional scan, 13 blocks were obtained in the healthy subjects and 10 in GL, totaling in 78 and 60 three-volume blocks per condition respectively.

fMRI data acquisition. A 1.5 T fMRI scanner (healthy subjects: Philips Intera; GL: Siemens Sonata) with a SENSE head coil (acceleration factor 1) was used to collect whole-brain anatomical scans using a highresolution T1-weighted anatomical protocol [healthy subjects and GL: repetition time (TR) $22 \mathrm{~ms}$, echo time (TE) $10 \mathrm{~ms}$, flip angle $30^{\circ}$, field of view (FOV) $256 \mathrm{~mm}^{2}$. Functional scans were acquired using a BOLD (blood oxygenation level-dependent) protocol and a $\mathrm{T} 2{ }^{*}$-weighted gradient-echo, echo-planar imaging (EPI) sequence (healthy subjects: flip angle $90^{\circ}$, TR $3.5 \mathrm{~s}$, TE $51 \mathrm{~ms}$, thickness $6 \mathrm{~mm}$, in-plane resolution $3.6 \times 3.6 \mathrm{~mm}$, FOV $256 \mathrm{~mm}^{2}$, matrix $64 \times 64$ voxels; GL: flip angle $90^{\circ}$, TR $4 \mathrm{~s}$, TE $51 \mathrm{~ms}$, thickness $4 \mathrm{~mm}$, in-plane resolution $4 \times 4 \mathrm{~mm}$, FOV $256 \mathrm{~mm}^{2}$, matrix $64 \times 64$ voxels). The scanning planes were oriented parallel to the line between the anterior and posterior commissure and covered the brain from the top of the cortex to the base of the cerebellum. In the healthy subjects, the functional data were reconstructed from an in-plane grid resolution of $64 \times 64$ to $128 \times 128$ voxels while still in frequency space.

fMRI data preprocessing. Using the Neurolens software package (www. neurolens.org; developed at the Neurovascular Imaging Laboratory, L'Unité de Neuroimagerie Fonctionnelle, Montreal, QC, Canada), all functional data were motion corrected with volume and scan three as reference. A general linear model (GLM) whole-brain analysis was performed on smoothed data (Gaussian full width at half-maximum filter of $6 \mathrm{~mm}$ ). A fixed effect model was used to generalize healthy subject activations to the group level. The resulting activation maps were thresholded to a false discovery rate (FDR) of $<0.01$.

The data were further preprocessed using custom-coded scripts in Matlab (The MathWorks) for subsequent multivariate analysis of insular activations. The data were linearly detrended, and, to offset hemodynamic delay and minimize within-trial variability, the first volume in each block was discarded and an average over the remaining two volumes was obtained (leaving a total of 78 volumes per stimulus for the healthy subjects and 60 for GL). The contralateral (left) insula, containing a subject mean of 827 (range 703-1004) voxels for the healthy participants and 297 voxels for GL, was subsequently identified manually using the Neurolens region of interest drawing tool under guidance of an anatomical reference (Naidich et al., 2004). Care was taken to minimize inclusion of voxels in the parietal operculum (second somatosensory cortex).

All analysis was performed in original individual space, and the resulting maps were transformed into Montreal Neurological Institute (MNI) standard stereotactic space (Evans et al., 1993) using SPM5 (Friston et al., 1994) with the supplied EPI brain as template. For data visualization, the programs MRIcron (by Chris Rorden, University of South Carolina, Columbia, SC, www.sph.sc.edu/comd/rorden/mricron/) and Cartool (by Denis Brunet, Functional Brain Mapping Laboratory, Geneva, Switzerland, http://brainmapping.unige.ch/Cartool.htm) were used.

The healthy subject data have previously been used for performance evaluation in a methodological paper describing the current analysis algorithm (Björnsdotter Åberg and Wessberg, 2008).

Multivariate analysis using evolutionary clustering. To investigate CT projection patterns in the insular cortex, we applied a multivariate clustering scheme based on pattern recognition concepts (Björnsdotter Åberg and Wessberg, 2008). The proposed method integrates discriminative modeling techniques with evolutionary optimization, aiming to identify clusters of voxels where a classifier can differentiate stimulus conditions. In the current study, the algorithm was used for identifying the one voxel cluster where two conditions are maximally separable, analogous to the GLM most activated voxels. For classification, we chose linear support vector machines (Suykens et al., 2002), popularly used in fMRI pattern recognition studies (Cox and Savoy, 2003; Mitchell et al., 2004; Kamitani and Tong, 2005; LaConte et al., 2005; Mourão-Miranda et al., 2005; De Martino et al., 2008), and the LS-SVMlab implementation for Matlab (developed by the Departement Elektrotechniek at Katholieke Universiteit Leuven, Leuven, Belgium).

The magnitude of condition separability (classification score) was quantified by the area under the receiver operating characteristic (ROC) curve (AUC). A value of 1 equals perfect separation and 0.5 corresponds to chance classification. Significance levels were established using nonparametric permutation testing (Siegel and Castellan, 1988).

Importantly, all reported classification scores were obtained on data partitions exclusively used for estimating the generalization ability of the final cluster with no part in the optimization process. Since evolutionary optimization is stochastic, as opposed to deterministic, in nature, the algorithm was iterated a number of times to establish robustly discriminative clusters.

The pattern recognition approach has numerous attractive properties 
in exploring cortical processing of complex stimuli (for review, see Haynes and Rees, 2006). Discriminative models require substantially fewer assumptions about the system under investigation than generative approaches such as the GLM. Notably, no explicit specification of the hemodynamic response function is necessary. Moreover, contrasting GLM voxel-by-voxel analysis, pattern recognition algorithms inherently use information encoded over multiple voxels to detect differences between conditions. This multivariate nature renders the algorithm more sensitive to condition differences than the GLM (Björnsdotter Åberg and Wessberg, 2008; De Martino et al., 2008). In addition, the approach requires no conventional spatial smoothing (which can, on the contrary, reduce performance because of inevitable information loss), and is thus superior in resolving small and distinct regions typical for somatotopy.

Importantly, the primary purpose of the present study was to use the multivariate algorithm for mapping regional responses to the brush stimulation and resolve a potential somatotopic organization, and no explicit attempts to obtain generalized classification predictions of single trials were made.

Region of interest identification. Previous studies have shown that, in addition to the highly activated posterior areas of the contralateral insular cortex, other insular regions also respond to soft brush stimulation (Olausson et al., 2002). Since the current algorithm performs most reliably in search spaces containing single optima, we decided to initially identify the insular region maximally responsive to generic brushing. Thus, in each subject, we applied the clustering scheme to the entire contralateral (left) insular cortex (as previously extracted, see fMRI data preprocessing for details) for the combined forearm and thigh brushing volumes contrasting the rest volumes. For improved classification performance, the datasets were balanced in terms of samples per condition category by upsamling the rest volumes to equal those of brushing. The algorithm was iterated 10 times and the voxel cluster size was optimized by the algorithm. The voxel cluster showing maximal differentiation (highest classification score) between brushing and rest was subsequently identified in each subject as a region of interest (ROI), to which further analysis was restricted.

Insular somatotopy exploration. To compare the forearm and thigh brushing projections, the clustering scheme was subsequently applied to the forearm/rest and thigh/rest datasets separately within the previously identified insular ROI. The cluster size was fixed to allow for direct comparison of classification performances within and between individuals. In GL, seven voxels was empirically determined to be a suitable cluster size for high classification performance. The corresponding volume in the healthy subjects, as a result of the higher spatial sampling frequency of the functional data, was 20 voxels. The algorithm was iterated 200 times, and the clusters which maximally differentiated the forearm and thigh stimuli from rest were obtained.

In each individual, the coordinates (in MNI space) of the forearm and thigh stimulation cluster centroids were identified. The healthy subject mean $(X, Y, Z)$ coordinates of the centroids were computed and significant differences between the forearm and thigh cluster locations determined using a paired $t$ test (two-tailed, $p<0.05$ ).

Cluster selectivity verification. Although the clustering scheme identifies regions maximally responsive to either forearm or thigh brushing, the possibility that any given cluster also responds to the other condition remains. Given a true somatotopic organization with consistent spatial separation of the conditions, the forearm data should be significantly less separable (lower AUC score) in the thigh cluster than in the forearm cluster (and the reverse for thigh stimuli). Also, the forearm data should be more separable than the thigh data in the forearm cluster (and vice versa). To verify the regional selectivity to forearm or thigh stimulation, we therefore removed the location bias by attempting classification of the two different conditions in both of the identified clusters. To establish reliable results, we performed a 10 -fold cross-validation, where the data (again, forearm/rest and thigh/rest) were divided into 10 sets of equal size. The linear support vector machine was trained on nine of the datasets, and the classification performance was obtained by applying the trained classifier to the tenth set. The classification scores reported here, again quantified in terms of AUC, reflect the average performance over the 10 folds.
Table 1. Peak $T$ values and MNI coordinates of whole-brain activations resulting from soft brushing on the forearm and the thigh for healthy subjects (group analysis) and the neuronopathy patient (GL), thresholded for a false discovery rate of $<0.01$

\begin{tabular}{llllll}
\hline & Forearm & & & Thigh \\
\cline { 2 - 3 } Region & Healthy & GL & & Healthy & GL \\
\hline S1 (contra) & $5.2(-26,-42,64)$ & n.s. & & $6.9(-16,-48,70)$ & n.s. \\
S2 (contra) & $6.6(-54,-26,20)$ & n.s. & & $8.3(-52,-28,20)$ & n.s. \\
S2 (ipsi) & $3.7(48,-26,24)$ & n.s. & & $6.4(54,-26,26)$ & n.s. \\
Other areas & BA6 & & & BA6, S1 face (ipsi) & n.s. \\
\hline
\end{tabular}

See Results, Insular somatotopy exploration, for local search results. BA, Brodmann area; contra, contralateral to the stimulation; ipsi, ipsilateral; n.s., non significant.

\section{Results}

\section{Psychophysics}

The neuronopathy patient (GL) could detect whether the forearm or thigh was stimulated in 31 out of 32 trials ( $97 \%$ correct), and she reported that she could clearly perceive the location of most of the stimuli. Although her performance appears surprisingly high, it should be pointed out that healthy subjects identify the stimulated limb without exception (Olausson et al., 2008a).

\section{Whole-brain analysis}

The localization of GLM peak activations for the healthy subjects (analyzed as a group) and the neuronopathy patient are summarized in Table 1. No significant activations were observed in the patient. There were, however, below-threshold tendencies for both forearm and thigh brushing in the contralateral posterior and midinsular cortex, consistent with previous observations ( $T>3$; but see Insular somatotopy exploration for a directed search of the insular cortex) (Olausson et al., 2002). In the healthy controls, there were, as expected, significant activations in the postcentral gyrus corresponding to the primary somatosensory cortex with a clear somatotopic organization (forearm brushing projecting more lateral and inferior than thigh brushing). Also, there were bilateral activations in the secondary somatosensory cortex with no distinct differences between forearm and thigh brushing. Although no activations were observed in the insular cortex on the group level, only one subject showed no significant activation $(T<3)$ attributable to any condition in a regional search on the individual level. In the remaining subjects, posterior to midinsular activations were found $(T>3$; but again, see Insular somatotopy exploration for a directed search) confirming our previous results (Olausson et al., 2002).

\section{Region of interest identification}

Within the contralateral (left) insular cortex, the cluster that maximally differentiated soft brushing from rest (region of interest for subsequent somatotopy analysis) was identified to the posterior region in all subjects, consistent with our previous observations (Olausson et al., 2002, 2008b). In GL, the cluster centroid was found at MNI $(X, Y, Z)$ coordinates $(-36,-16,6)$, and the corresponding location for the healthy subject mean centroid was nearby at $(-36,-18,6)$. The locations of the identified clusters are illustrated in Figure 1.

The condition discrimination performance of the identified cluster was highly significant in GL with an AUC of 0.74 and a cluster size of 97 voxels ( $p<0.05$, permutation test), as well as for the healthy volunteers at a subject mean AUC of 0.81 (range $0.75-0.85$ ) for a cluster size of 222 (range 177-261) voxels $(p<$ 0.05 , permutation test). 

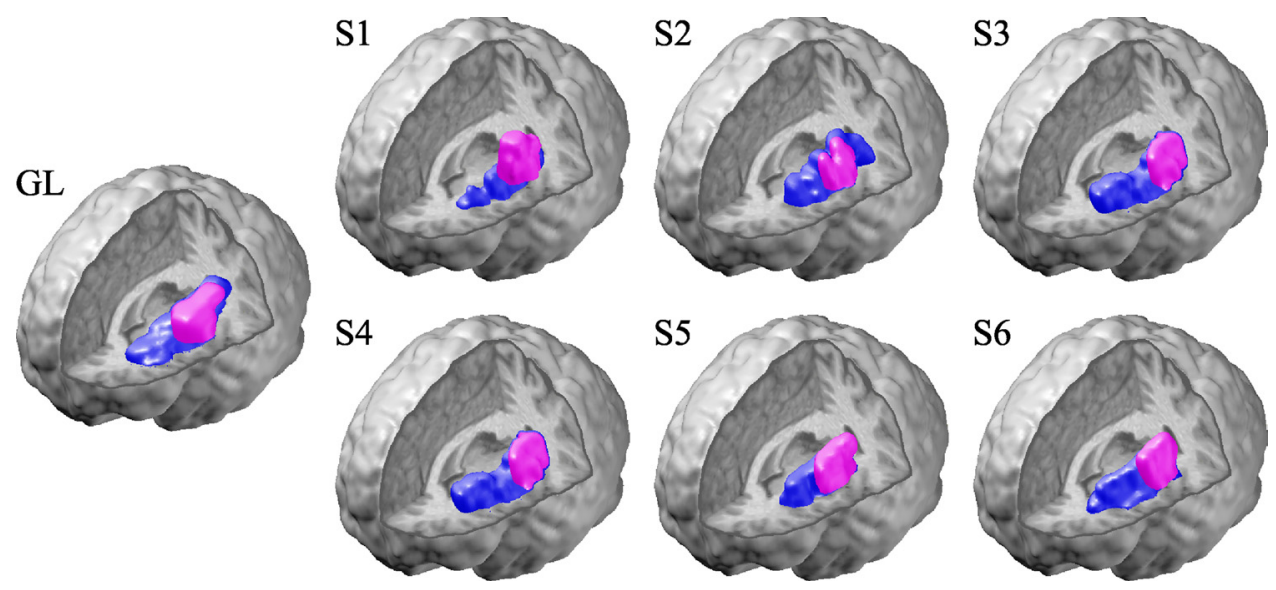

Figure 1. Insular region of interest. Voxel clusters maximally differentiating a soft brush stimulation from rest (purple) in the neuronopathy syndrome patient GL (lacking thick, myelinated afferents) and six healthy subjects (S1-S6). The brushing stimulation analysis was restricted to the manually identified contralateral (left) insular cortex (blue). Note that the response is consistently located to the posterior insular cortex across all subjects, including GL, in line with our previous observations (Olausson et al., 2002, 2008).
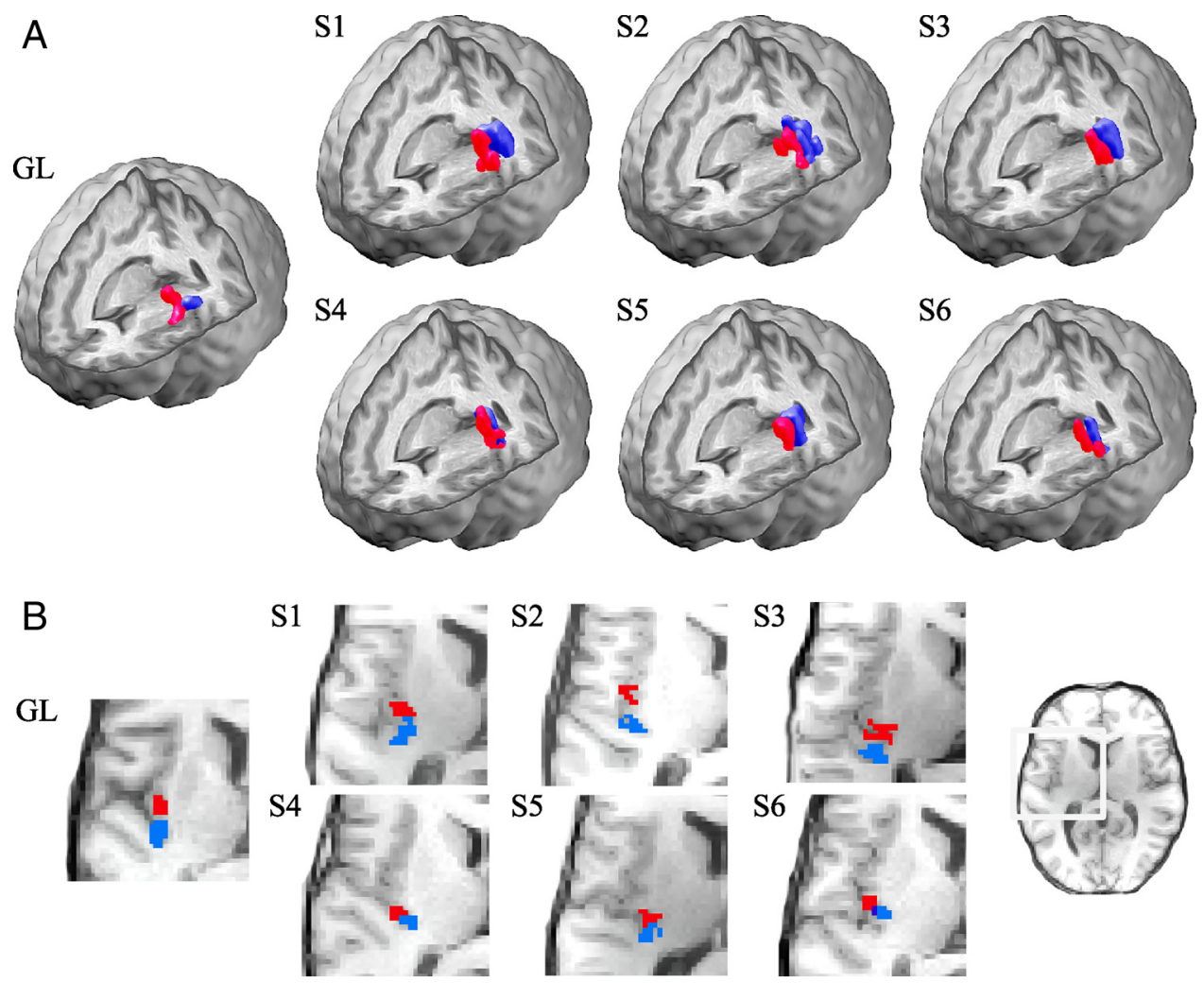

Figure 2. Insular somatotopy in response to gentle touch. $A$, The posterior contralateral (left) insular regions maximally differentiating forearm (red) and thigh (blue) soft brush stimulation from rest in the neuronopathy syndrome patient GL and six healthy subjects (S1-S6). Cluster overlap is shown in dark blue. The somatotopic organization was consistently identified in all subjects, with forearm brushing projecting anterior to thigh brushing. $\boldsymbol{B}$, Horizontal slices (MNIZ =6) of the same clusters, highlighting the anterior-posterior distinction.

\section{Insular somatotopy exploration}

Forearm and thigh tactile stimulation were found to project to distinctly separate locations in the posterior insular cortex in GL, with a substantial Euclidean distance between cluster centroids of $8.9 \mathrm{~mm}$ (Fig. 2). The forearm cluster centroid was located at MNI $(X, Y, Z)$ coordinates $(-34,-10,4)$, and the thigh cluster was found at $(-34,-18,0)$. The distance between clusters was thus maximal in the anterior-posterior $(Y)$ plane at $8 \mathrm{~mm}$, whereas the location differences in the remaining planes were nonexistent or small (X: $0 \mathrm{~mm}, Z: 4 \mathrm{~mm}$ ). There was a two (out of seven) voxel overlap between the clusters.
Validating the pattern observed in GL, the healthy subject insular projections were also arranged in a distinct somatotopic manner. As Figure 2 illustrates, the body-map organization with forearm brushing (red) projecting anterior to that of thigh (blue) was clear, both in the whole-brain rendering $(2 \mathrm{~A})$ and the detailed two-dimensional axial slices (2B). The subject mean Euclidean distance between the cluster centroids equaled that of GL at 9.3 (range $6.6-12) \mathrm{mm}$, whereas the overlap was substantially smaller at 0.83 (out of 20 ) voxels. The mean ( \pm SD) MNI coordinates of the forearm stimulation cluster centroid were $(-37 \pm$ $3.5,-17 \pm 4.8,6.2 \pm 6.6)$, whereas the thigh brushing cluster was 
found at $(-36 \pm 3.1,-25 \pm 4.1,7.7 \pm$ 5.9). Thus, GL, being within two SDs of the healthy subject values, does not differ significantly from the norm. The individual forearm and thigh cluster centroid locations are shown in Figure $3 A$, again illustrating the consistency in activation pattern across all individuals including GL. The difference in location [healthy subject mean $(-0.83 \pm 2.0,7.3 \pm 3.0,-1.5 \pm 5.4$ $\mathrm{mm}$ ) relative to the thigh centroid] was significant only in the $Y$-plane (anteriorposterior; two-tailed paired $t$ test, $p<0.05$ ), which is also clear from the MNI coordinate scatter plots of individual centroid displacements (forearm centroid location minus thigh centroid location) in Figure $3 B$.

\section{Cluster selectivity verification}

The cluster selectivity verification procedure showed that the anterior clusters were indeed more selective to forearm than thigh stimulation and vice versa (Fig. 4). The healthy subject forearm brushing volumes were significantly more separable in the anterior cluster (subject mean $\mathrm{AUC}=0.71)$ than in the posterior cluster (subject mean AUC $=0.62$; two-tailed paired $t$ test, $p<0.05)$ and vice versa for thigh brushing (subject mean AUC $=0.60$ and AUC $=0.69$, respectively; $p<0.05$ ). Similarly, the forearm brushing volumes were significantly more separable than those of thigh brushing in the anterior cluster, and vice versa $(p<0.05)$. Following the healthy subject classification scores, the neuronopathy patient forearm brushing volumes were more separable in the anterior cluster $(\mathrm{AUC}=0.65)$ than in the posterior cluster $(A U C=0.62)$ and vice versa for the thigh brushing volumes (AUC $=0.59$ and $\mathrm{AUC}=0.70$ in the anterior and posterior clusters respectively).

\section{Discussion}

We have demonstrated that contralateral posterior insular cortex processing of gentle tactile stimulation is organized in a somatotopic fashion, such that soft brushing of the forearm projects anterior to brushing of the thigh. The somatotopy was observed not only in healthy individuals but also in a neuronopathy patient (GL) lacking thick, myelinated $\mathrm{A} \beta$ fibers, indicating that the activation pattern reflects central projections of small-diameter CT afferents. Moreover, the observed body-map arrangement is consistent with the patient's crude ability to localize soft brush tactile stimuli in a forced-choice situation.

\section{Afferent activation}

The soft brush stimulus used here vigorously activates CT afferents, as demonstrated in human microneurography recordings (Vallbo et al., 1999, Löken et al., 2009). Nevertheless, the concurrent activation of rapidly conducting myelinated $(\mathrm{A} \beta)$ fibers is inevitable in healthy subjects. The consistency in insular activation patterns between the group of healthy individuals and the neuronopathy subject (lacking $\mathrm{A} \beta$ afferents) does, however, suggest that the observed somatotopic organization indeed reflects central projections of CT afferents rather than of myelinated fibers. Moreover, the activation consistency suggests that neither
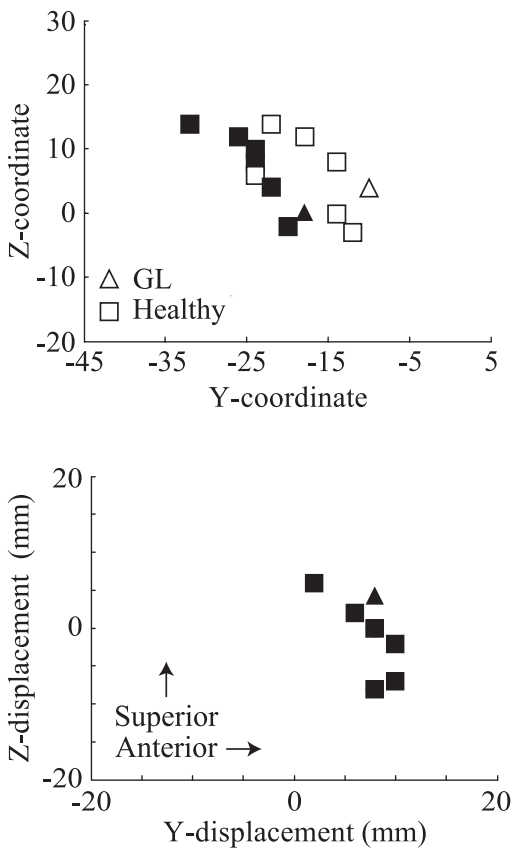

0

Figure 3. Forearm and thigh projections in response to gentle touch. $\boldsymbol{A}, \boldsymbol{B}$, The forearm (white) and thigh (black) cluster neuronopathy syndrome patient GL (triangles) clearly illustrate the observed somatotopic organization of the contralateral (left) posterior insular cortex. The healthy subjects showed a significant difference between forearm and thigh cluster centroid location in the $Y$-plane only, with forearm located anterior to thigh stimulation (two-tailed paired $t$ test, $p<0.05$ ).

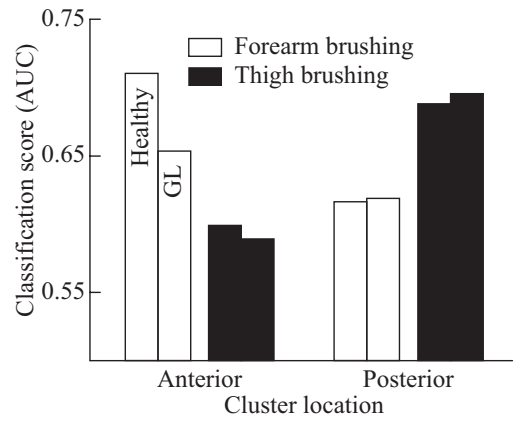

Figure 4. Cluster selectivity verification. The classification scores (measured by AUC, where a value of 1 equals perfect separation) of the forearm (white) and thigh (black) brushing volumes on the posterior insular voxels obtained using the clustering algorithm (as seen in Fig. 2). The healthy subject (left bar) forearm brushing volumes were significantly more separable in the forearm cluster than in the thigh cluster, and vice versa for the thigh brushing volumes (twotailed paired $t$ test, $p<0.05$ ). Also, the forearm brushing scores were significantly higher than those of thigh brushing in the anterior cluster, and vice versa (two-tailed paired $t$ test, $p<$ 0.05). The neuronopathy patient data (GL; right bar) follow the healthy subject trend. These results verify the forearm-anterior and thigh-posterior pattern demonstrated by the clustering algorithm.

the age difference between the healthy subjects (aged 22-28 years) and GL (56 years) nor the slight variations in experimental protocol have influenced the results.

\section{Central organization of CT afferents}

We and others have previously speculated that CT afferents are organized in a manner similar to that of the pain- and temperature-mediating thin-fiber systems projecting through the lamina I spinothalamic pathway to the insular cortex (Olausson et al., 2002) (for a review of the pathway, see Craig, 2002). Lamina I contains modality-specific classes of neurons receiving 


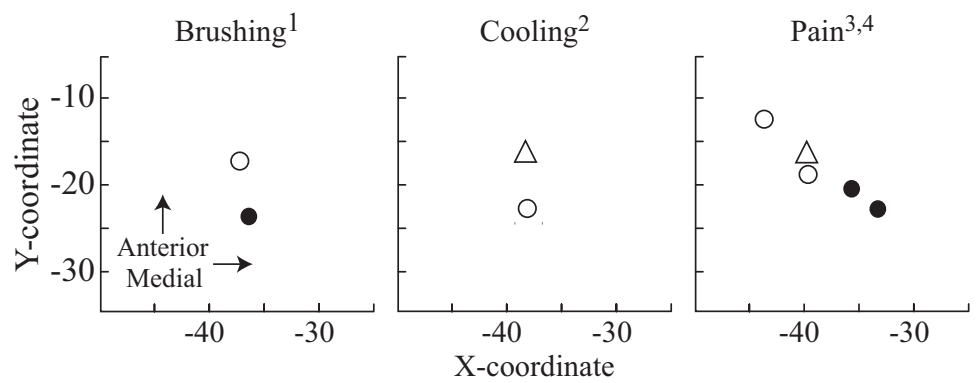

$\triangle$ Neck/face $\bigcirc \mathrm{Arm} / \mathrm{hand} \bullet$ Thigh/foot

Figure 5. Thin-fiber activation center comparison. Posterior insular cortex activation centers (in MNI coordinates) resulting from application of thin-fiber exciting stimuli (soft brushing, innocuous cooling, and pain) on various body parts reported in the present ${ }^{1}$ and previous studies (Hua et al., $2005^{2}$, Henderson et al., $2007^{3}$; Brooks et al., 2005 ${ }^{4}$ ). White markers indicate an upper body stimulus location and black a lower body location. Gentle tactile stimulation (brushing) fit the somatotopic pattern in the posterior contralateral insular cortex well, with upper body stimulations projecting anterior (and slightly lateral) to lower body stimulations. The nature of the relative spatial organization of these modalities within the posterior insular cortex has yet to be demonstrated in humans, however.

input from functionally distinct groups of small-diameter afferents (Han et al., 1998; Craig et al., 2001), including those excited by gentle mechanical stimuli (Kumazawa and Perl, 1977; Sugiura et al., 1986; Light and Willcockson, 1999). Lamina I neurons responding to noxious and temperature stimuli, in turn, project somatotopically through a distinct nucleus of the posterior ventromedial thalamus (VMpo) (Craig et al., 1994; Dostrovsky and Craig, 1996) (but see Willis et al., 2002) to the posterior insular cortex (Craig, 1995; Brooks et al., 2005; Hua le et al., 2005; Henderson et al., 2007). The presently observed insular activation pattern resulting from CT excitation, highly similar to that of painful and cooling stimuli as shown in Figure 5, supports the notion that CT and other thin-fiber afferents indeed project along the lamina I spinothalamic pathway to the posterior insular cortex. The relative spatial arrangement of these distinct modalities in the dorsal posterior insula remains to be explored, however.

The thin-fiber system is, moreover, proposed not only as an afferent homeostatic pathway, but also to contribute significantly to the construction of the subjective experience of the self and awareness (Craig, 2009). The neuronopathy patient, although denying a general sense of touch, could perceive as well as localize the soft brush stimulation in the experimental situation. Whereas the somatotopically organized posterior insular cortex serves as a primary interoceptive region, the reported subsequent mid-toanterior progression of integration of various physiological representations is likely to play a substantial role in generating an awareness of the tactile stimulation (Burton et al., 1993; Spinazzola et al., 2008; Craig, 2009). Indeed, the regional search in the patient as well as in a number of the healthy subjects revealed activation of the mid-insula. In addition, we have previously observed that soft brushing induces robust activity in the anterior insular cortex (Olausson et al., 2002), implied as integral in the subjective evaluation of the body's condition ("how you feel") (Craig, 2009). None of such was, however, found in the current study, possibly since the subjects were not instructed to evaluate the soft brush stimulation experience. Also, it should be noted that the current study focused on the somatosensory and insular cortices, and additional networks are likely involved.

\section{CT system function}

The system of thin-fiber afferents is considered to play a fundamental role in interoception (the sense of the physiological condition of the body) and, thus, homeostasis (Craig, 2002). We, and others, have previously proposed that the CT network relates to emotional and social, as opposed to discriminative, aspects of tactile stimulation (Vallbo et al., 1993, 1999; Essick et al., 1999; Olausson et al., 2002), and we recently demonstrated that CT afferents signal pleasant touch in healthy humans (Löken et al., 2009). In this light, the CT system contributes to the maintenance of physical integrity and well-being (that is, homeostasis) by relaying information regarding the affective tactile status of the body, as opposed to thickfiber-mediated touch relevant for various cognitive behaviors such as texture discrimination. The tactile well-being of the body is of fundamental importance, not the least in primates, as illustrated in the classic study of infant monkeys displaying affection for a surrogate mother in response to tactile comfort (Harlow, 1958). In fact, we argue that the CT system provides an important sensory underpinning of social behavior and, moreover, plays an integral role in the foundation of self-awareness (McGlone et al., 2007; Olausson et al., 2008c; Craig, 2009).

Although the $\mathrm{A} \beta$ system is clearly dominant in the accurate localization of tactile stimuli, our study suggests that there is also a crude sensory-discriminatory functionality to the CT system. Consistent with the somatotopic organization in the insular cortex, the neuronopathy subject, despite lacking thick myelinated afferents and denying any ability of sensing touch below the level of the nose in daily life, could localize the soft brush stimulation to the thigh or arm at an accuracy of $97 \%$ in the forced-choice situation (and $72 \%$ in a previous quadrant study) (Olausson et al., 2008a). The contrast compared with neurologically intact individuals is, however, striking - healthy subjects can localize point indentation on hairy skin with an accuracy of $\sim 2 \mathrm{~cm}$ (Norrsell and Olausson, 1994). Also, the patient's CT system potentially serves an amplified discriminative function resulting from central sensory representation adaptations, although, given the similarity in brain activation pattern with the healthy controls, it is highly unlikely that the insular somatotopy reflects such neuroplastic changes.

Nonetheless, it appears improbable that the CT system plays a significant role in acute spatial localization. Yet, it can be presumed that the general stimulus location significantly modulates affective sensations, which, as opposed to $\mathrm{A} \beta$ mediated percepts, are intimately related to CT activity (Löken et al., 2009). Propagation of such affective information is fundamentally important in the design or preparation of appropriate actions in response to emotionally relevant stimuli. For example, it has been shown in rats, cats, and humans that painful stimuli applied to various body parts result in correspondingly different autonomic responses (again related to homeostasis) (Lewis, 1942; Bandler et al., 2000). It can thus be hypothesized that the crude localization capacity of the CT system serves a similar function, where, for example, a gentle stroke on the cheek evokes a different emotional and motivational response than that on the arm, thus signaling various affective aspects with corresponding social implications.

\section{References}

Bandler R, Price JL, Keay KA (2000) Brain mediation of active and passive emotional coping. Prog Brain Res 122:333-349.

Bessou P, Burgess PR, Perl ER, Taylor CB (1971) Dynamic properties of mechanoreceptors with unmyelinated (C) fibers. J Neurophysiol 34:116-131. 
Björnsdotter Åberg M, Wessberg J (2008) An evolutionary approach to the identification of informative voxel clusters for brain state discrimination. IEEE J Select Topics Signal Proc 2:919-928.

Blomqvist A, Craig AD (2000) Is neuropathic pain caused by the activation of nociceptive-specific neurons due to anatomic sprouting in the dorsal horn? J Comp Neurol 428:1-4.

Brooks JC, Zambreanu L, Godinez A, Craig AD, Tracey I (2005) Somatotopic organisation of the human insula to painful heat studied with high resolution functional imaging. Neuroimage 27:201-209.

Burton H, Videen TO, Raichle ME (1993) Tactile-vibration-activated foci in insular and parietal-opercular cortex studied with positron emission tomography: mapping the second somatosensory area in humans. Somatosens Mot Res 10:297-308.

Cox DD, Savoy RL (2003) Functional magnetic resonance imaging (fMRI) 'brain reading': detecting and classifying distributed patterns of fMRI activity in human visual cortex. Neuroimage 19:261-270.

Craig AD (1995) Supraspinal projections of lamina I neurons in forebrain areas involved in pain processing, Ed 2. New York: Libbey Eurotext Montrouge.

Craig AD (2002) How do you feel? Interoception: the sense of the physiological condition of the body. Nat Rev Neurosci 3:655-666.

Craig AD (2003) Pain mechanisms: labeled lines versus convergence in central processing. Ann Rev Neurosci 26:1-30.

Craig AD (2009) How do you feel - now? The anterior insula and human awareness. Nat Rev Neurosci 10:59-70.

Craig AD, Zhang ET (2006) Retrograde analyses of spinothalamic projections in the macaque monkey: input to posterolateral thalamus. J Comp Neurol 499:953-964.

Craig AD, Bushnell MC, Zhang ET, Blomqvist A (1994) A thalamic nucleus specific for pain and temperature sensation. Nature 372:770-773.

Craig AD, Zhang ET, Blomqvist A (1999) A distinct thermoreceptive subregion of lamina I in nucleus caudalis of the owl monkey. J Comp Neurol 404:221-234.

Craig AD, Chen K, Bandy D, Reiman EM (2000) Thermosensory activation of insular cortex. Nat Neurosci 3:184-190.

Craig AD, Krout K, Andrew D (2001) Quantitative response characteristics of thermoreceptive and nociceptive lamina I spinothalamic neurons in the cat. J Neurophysiol 86:1459-1480.

De Martino F, Valente G, Staeren N, Ashburner J, Goebel R, Formisano E (2008) Combining multivariate voxel selection and Support Vector Machines for mapping and classification of fMRI spatial patterns. Neuroimage 43:44-58.

Dostrovsky JO, Craig AD (1996) Cooling-specific spinothalamic neurons in the monkey. J Neurophysiol 76:3656-3665.

Douglas WW, Ritchie JM (1957) Nonmedullated fibres in the saphenous nerve which signal touch. J Physiol 139:385-399.

Edin B (2001) Cutaneous afferents provide information about knee joint movements in humans. J Physiol 531:289-297.

Essick GK, James A, McGlone FP (1999) Psychophysical assessment of the affective components of non-painful touch. Neuroreport 10:2083-2087.

Evans AC, Collins DL, Mills SR, Brown ED, Kelly RL, Peters TM (1993) 3D statistical neuroanatomical models from 305 MRI volumes. Paper presented at the IEEE Nuclear Science Symposium and Medical Imaging Conference, San Francisco, CA, November.

Forget R, Lamarre Y (1995) Postural adjustments associated with different unloadings of the forearm: effects of proprioceptive and cutaneous afferent deprivation. Can J Physiol Pharmacol 73:285-294.

Friston KJ, Holmes AP, Worsley KJ, Poline JP, Frith CD, Frackowiak RSJ (1994) Statistical parametric maps in functional imaging: a general linear approach. Hum Brain Mapp 2:189-210.

Han ZS, Zhang ET, Craig AD (1998) Nociceptive and thermoreceptive lamina I neurons are anatomically distinct. Nat Neurosci 1:218-225.

Harlow HF (1958) The nature of love. Am Psychol 13:673-685.

Haynes JD, Rees G (2006) Decoding mental states from brain activity in humans. Nat Rev Neurosci 7:523-534.

Henderson LA, Gandevia SC, Macefield VG (2007) Somatotopic organization of the processing of muscle and cutaneous pain in the left and right insula cortex: A single-trial fMRI study. Pain 128:20-30.

Hua le H, Strigo IA, Baxter LC, Johnson SC, Craig AD (2005) Anteroposterior somatotopy of innocuous cooling activation focus in human dorsal posterior insular cortex. Am J Physiol Regul Integr Comp Physiol 289:R319-R325.

Iggo A, Muir AR (1969) The structure and function of a slowly adapting touch corpuscle in hairy skin. J Physiol 200:763-796.
Kamitani Y, Tong F (2005) Decoding the visual and sub jective contents of the human brain. Nat Neurosci 8:679-685.

Kumazawa T, Perl ER (1977) Primate cutaneous sensory units with unmyelinated (C) afferent fibers. J Neurophysiol 40:1325-1338.

LaConte S, Strother S, Cherkassky V, Anderson J, Hu X (2005) Support vector machines for temporal classification of block design fMRI data. Neuroimage 26:317-329.

Lewis T (1942) Pain. New York: MacMillan.

Light AR, Willcockson HH (1999) Spinal laminae I-II neurons in rat recorded in vivo in whole cell tight seal configuration: properties and opioid responses. J Neurophysiol 82:3316-3326.

Löken LS, Wessberg J, Olausson H (2007) Unmyelinated tactile (CT) afferents are present in the human peroneal and radial nerves. Soc Neurosci Abstr 37:827.2.

Löken LS, Wessberg J, Morrison I, McGlone F, Olausson H (2009) Coding of pleasant touch by unmyelinated afferents in humans. Nat Neurosci 12:547-548.

McGlone F, Vallbo AB, Olausson H, Löken LS, Wessberg J (2007) Discriminative touch and emotional touch. Can J Exp Psychol 61:173-183.

Mitchell TM, Hutchinson R, Niculescu RS, Pereira F, Wang X, Just M, Newman S (2004) Learning to decode cognitive states from brain images. Mach Learn 57:145-175.

Mourão-Miranda J, Bokde AL, Born C, Hampel H, Stetter M (2005) Classifying brain states and determining the discriminating activation patterns: support vector machine on functional MRI data. Neuroimage 28:980-995.

Naidich TP, Kang E, Fatterpekar GM, Delman BN, Gultekin SH, Wolfe D, Ortiz O, Yousry I, Weismann M, Yousry TA (2004) The insula: anatomic study and MR imaging display at 1.5 T. Am J Neuroradiol 25:222-232.

Nordin M (1990) Low-threshold mechanorecepetive and nociceptive units with unmyelinated C fibres in the human supraorbital nerve. J Physiol 426:229-240

Norrsell U, Olausson H (1994) Spatial cues serving the tactile directional sensibility of the human forearm. J Physiol 478:533-540.

Olausson H, Lamarre Y, Backlund H, Morin C, Wallin BG, Starck G, Ekholm S, Strigo I, Worsley K, Vallbo AB, Bushnell MC (2002) Unmyelinated tactile afferents signal touch and project to insular cortex. Nat Neurosci 5:900-904.

Olausson H, Cole J, Rylander K, McGlone F, Lamarre Y, Wallin BG, Krämer H, Wessberg J, Elam M, Bushnell MC, Vallbo AB (2008a) Functional role of unmyelinated tactile afferents in human hairy skin: sympathetic response and perceptual localization. Exp Brain Res 184:135-140.

Olausson HW, Cole J, Vallbo A, McGlone F, Elam M, Krämer HH, Rylander K, Wessberg J, Bushnell MC (2008b) Unmyelinated tactile afferents have opposite effects on insular and somatosensory cortical processing. Neurosci Lett 436:128-132.

Olausson H, Wessberg J, Morrison I, McGlone F, Vallbo AB (2008c) The neurophysiology of unmyelinated tactile afferents. Neurosci Biobehav Rev. Advance online publication. Retrieved Oct. 8, 2008. doi: 10.1016/j.neubiorev.2008.09.011.

Siegel S, Castellan NJ Jr (1988) Nonparametric statistics for the behavioral sciences, Ed 2. New York: McGraw-Hill.

Spinazzola L, Pia L, Folegatti A, Marchetti C, Berti A (2008) Modular structure of awareness for sensorimotor disorders: evidence from anosognosia for hemiplegia and anosognosia for hemianaesthesia. Neuropsychologia 46:915-926.

Sterman AB, Schaumburg HH, Asbury AK (1980) The acute sensory neuronopathy syndrome: a distinct clinical entity. Ann Neurol 7:354-358.

Sugiura Y, Lee CL, Perl ER (1986) Central projections of identified unmyelinated (C) afferent fibers innervating mammalian skin. Science 234:358-361.

Suykens JAK, Van Gestel T, De Brabanter J, De Moor B, Vandewalle J (2002) Least squares support vector machines. Singapore: World Scientific.

Vallbo A, Olausson H, Wessberg J, Norrsell U (1993) A system of unmyelinated afferents for innocuous mechanoreception in the human skin. Brain Res 628:301-304.

Vallbo AB, Olausson H, Wessberg J (1999) Unmyelinated afferents constitute a second system coding tactile stimuli of the human hairy skin. J Neurophysiol 81:2753-2763.

Willis WD Jr, Zhang X, Honda CN, Giesler GJ Jr (2002) A critical review of the role of the proposed VMpo nucleus in pain. J Pain 3:79-94.

Zotterman Y (1939) Touch pain and tickling: an electro-physiological investigation on cutaneous sensory nerves. J Physiol 95:1-28. 